\title{
Aligned Heterostructures of Single-Crystalline Tin Nanowires Encapsulated in Amorphous Carbon Nanotubes
}

\author{
Ruying Li, ${ }^{\dagger}$ Xiangcheng Sun, ${ }^{\dagger}$ Xiaorong Zhou,, Mei Cai, ${ }^{\S}$ and Xueliang Sun $*, \dagger$ \\ Department of Mechanical and Materials Engineering, University of Western Ontario, London, \\ Ontario, Canada, N6A 5B9, School of Materials, The University of Manchester, Manchester, \\ United Kingdom, M60 1QD, and General Motors Research and Development Center, \\ Warren, Michigan 48090-9055
}

Received: January 28, 2007; In Final Form: April 24, 2007

\begin{abstract}
High-density heterostructures of amorphous carbon nanotubes encapsulated single-crystalline tin nanowires (a-CNT-Sn) have successfully been synthesized by using the simple thermal evaporation of solid tin powder (Sn) and introduction of ethylene gas $\left(\mathrm{C}_{2} \mathrm{H}_{4}\right)$. A commercially widely used porous carbon paper, consisting of micrographitic fibers, was employed as growing substrate. The morphology, composition, and structure of the synthesized nano-heterostructures were examined using scanning electron microscopy (SEM), transmission electron microscopy (TEM), and energy-dispersive X-ray (EDX) analysis. It was found that the resultant heterostructures are uniformly distributed on graphitic fibers and that these well-defined tin $(\mathrm{Sn})$ nanowires, up to $10 \mu \mathrm{m}$ in length, are completely covered with amorphous carbon walls of about $30 \mathrm{~nm}$ thickness. The hollow features at the tips of such heterostructures were revealed. A growth mechanism has been proposed to explain the growth processes for such novel heterostructures. The synthesis method reported here can be employed to fabricate oriented nanotube arrays filled with other nanowires on a variety of substrates at large scale.
\end{abstract}

\section{Introduction}

Over the past decade, carbon nanotubes (CNTs), because of their unique structures, have attracted significant research efforts. CNTs possess special properties and have tremendous potential for applications in composite materials, electrode materials, field emitters, nanoelectronics, and nanosensors. ${ }^{1}$ Metal and semiconductor nanowires (NWs) have also been developed as complementary to CNTs. ${ }^{2}$ Further, considerable effort has been made to explore various heterostructures with the combination of CNTs and NWs. Such one-dimensional (1D) heterostructures are of significance to both fundamental studies in nanoscience and potential industrial applications in nanotechnology. ${ }^{3-5}$ Examples of such heterostructures of carbon nanotube encapsulated nanowires include $\mathrm{CNTs}-\mathrm{Ni},{ }^{3 \mathrm{~d}} \mathrm{CNTs}-\mathrm{Ag},{ }^{4 \mathrm{a}} \mathrm{CNTs}-$ $\mathrm{SnO}_{2},{ }^{4 \mathrm{c}} \mathrm{CNTs}-\mathrm{ZnO},{ }^{5 \mathrm{a}}$ and $\mathrm{CNTs}-\mathrm{ZnS} .{ }^{5 \mathrm{c}}$ Synthesis methods for such carbon nanotube-nanowire heterostructures usually fall into two categories: (1) chemical vapor deposition ${ }^{3,5 a, 5 b}$ and (2) anodic alumina membrane (AAM) template-based method. ${ }^{4}$

In the above-mentioned works, CNTs encapsulated metal nanowires, with perfect concentric graphite shells, were successfully synthesized. However, for many applications, the surfaces of CNTs have to be modified to introduce "defects" in the carbon network. ${ }^{6}$ Amorphous carbon nanotubes, meanwhile, have displayed interesting semiconducting characteristics that promise potential for applications in nanoelectronics and nanosensors. ${ }^{4 a}$ Therefore, various methods have been recently developed to produce amorphous CNTs. ${ }^{7}$ In addition, Sn-based nanostructures are also of special interest because of their

* To whom correspondence should be addressed. Phone: 519-661-2111 ext. 87759. Fax: 519-661-3020. E-mail: xsun@eng.uwo.ca.

University of Western Ontario.

$\doteqdot$ The University of Manchester.

$\S$ General Motors Research and Development Center. potential for applications in the area of gas sensors, Li-ion storage for batteries, and superconductors. ${ }^{3 \mathrm{c}, 4 \mathrm{c}}$ An example of Sn-based nanostructure is small $\mathrm{Sn}$ particles $(\sim 100 \mathrm{~nm})$ contained in amorphous carbon hollow spheres $(100-1000 \mathrm{~nm})$, which were suggested as a potential solution to the capacityfading problem of Sn-based lithium storage compounds. ${ }^{8}$ To date, several tin oxide $\left(\mathrm{SnO}_{2}\right.$ and $\left.\mathrm{SnO}\right)$ nanowires and nanobelts have been successfully synthesized. ${ }^{9}$ However, less information can be found in the open literature on the formation of metallic tin nanowires. ${ }^{10}$ In particular, large-scale synthesis of amorphous carbon nanotubes encapsulated tin nanowires remains an even greater challenge.

In this communication, we report the first synthesis of highdensity, aligned heterostructures, consisting of amorphous carbon nanotubes and metallic tin nanowires, on graphitic fibers by using thermal evaporation of tin powder under an atmosphere of ethylene $\left(\mathrm{C}_{2} \mathrm{H}_{4}\right)$ and argon gas (Ar) mixture at $900{ }^{\circ} \mathrm{C}$. Such heterostructure formed on commercially widely used carbon paper might find significant applications as electrodes for sensors and fuel cells.

\section{Experimental Procedures}

Growth of Amorphous Carbon Nanotubes Encapsulated Sn Nanowires. The heterostructures were synthesized by thermal evaporation method. In a typical procedure, pure commercial grade Sn powders (2 g, -325 mesh, 99.8\%) were loaded in an alumina boat placed at the middle of a quartz tube in a horizontal tube furnace. A small piece of commercially available carbon paper was placed beside Sn powder, which acted as a substrate for the growth of products. The reaction chamber was heated from room temperature to $900{ }^{\circ} \mathrm{C}$ (in about $15 \mathrm{~min}$ ) under an atmosphere of flowing $\mathrm{Ar}$ and $2 \%$ ethylene $(200 \mathrm{sccm})$. The most important feature of our method 

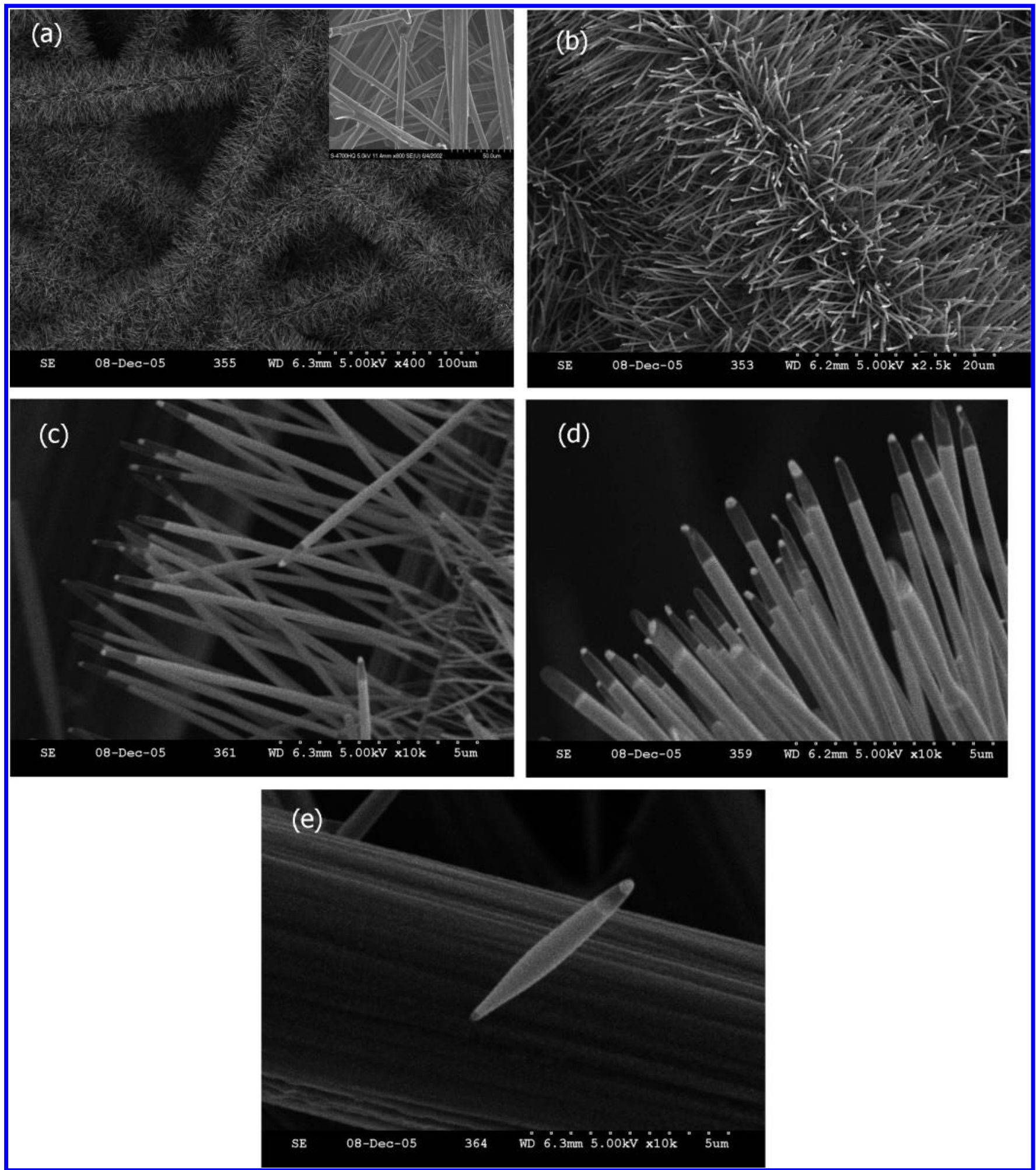

Figure 1. (a) SEM images showing the morphologies of high-density carbon nanotubes encapsulating pure Sn nanowires grown on graphitic fibers; inset: bare graphitic fibers on carbon paper. (b) Higher magnification SEM images showing radical nanostructures along a graphitic fiber; (c, d) increased magnification SEM images showing the tip of nanostructure array; (e) an individual nanostructure on graphitic fiber.

is the simultaneous introduction of $\mathrm{C}_{2} \mathrm{H}_{2}$ gas into the reaction system. Subsequently, the furnace was kept at $900{ }^{\circ} \mathrm{C}$ for $2 \mathrm{~h}$ and then was cooled to room temperature. After the reaction, it was observed that gray darklike products (thin films) were deposited on the surface of the carbon paper substrates. In this work, no carbon (i.e., carbon black, carbon nanotubes, graphite, etc.) was mixed into the starting Sn powders, and no metal catalyst was predeposited on carbon paper substrate.
Product Characterization. The synthesized product was examined initially using Hitachi S-2600N scanning electron microscopy (SEM), equipped with energy dispersive X-ray (EDX) analysis facility. Further characterization of the detailed 1-D nanostructures was carried out using a JEOL 2010 FEG transmission electron microscope (TEM) at $200 \mathrm{kV}$. The crystal structure of the produced nanostructures was determined by high-resolution transmission electron microscopy and selected area electron diffraction in a Tecnai $\mathrm{G}^{2}$ F30 TEM. 

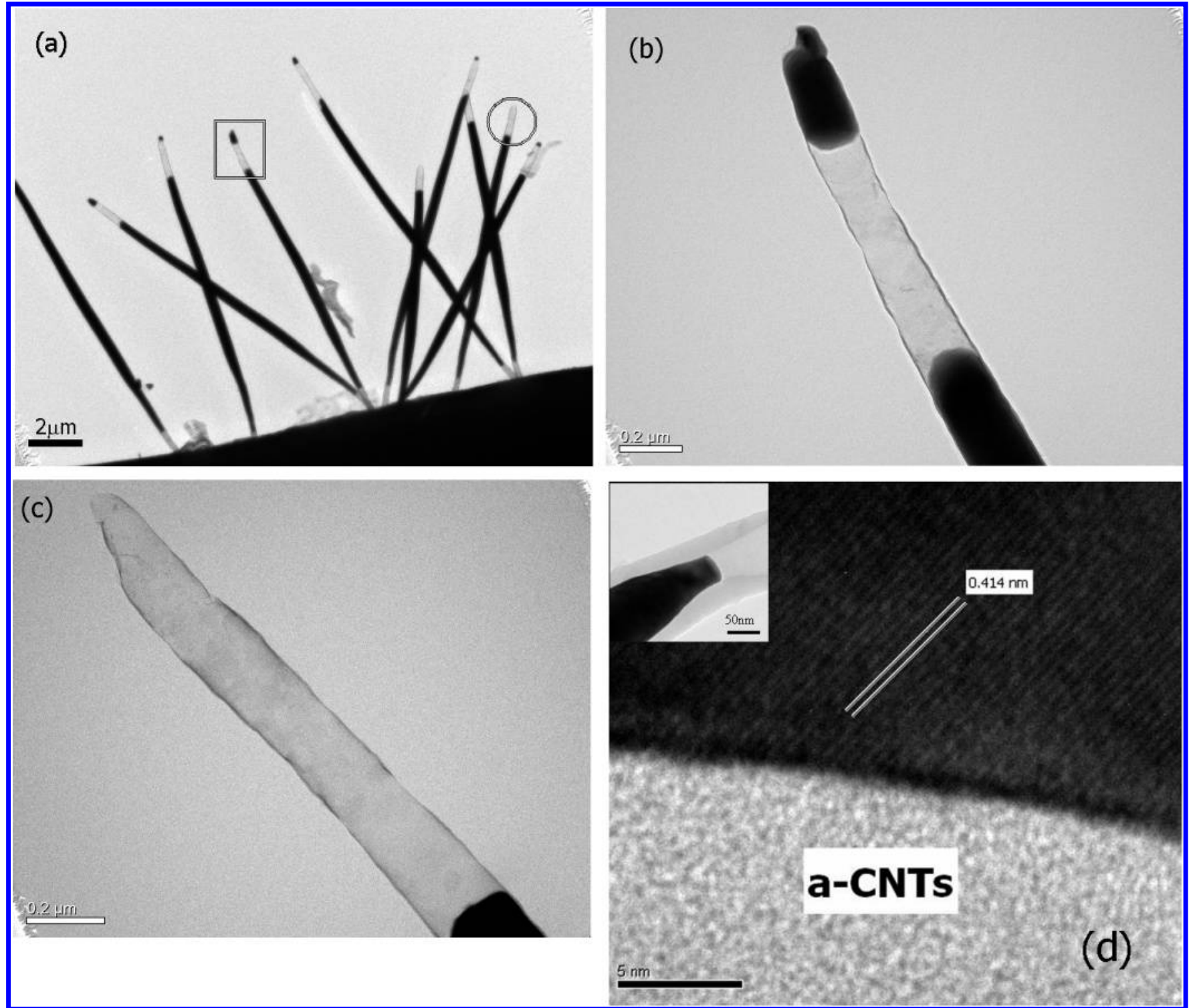

Figure 2. (a) TEM images of several individual nanostructures showing carbon nanotubes encapsulating Sn nanowires; (b, c) increased magnification images of the tips of individual nanostructure, revealing the hollow region located at each tip; and (d) high-resolution TEM image showing the lattice image of Sn core and amorphous carbon shell; inset is the typical encapsulation of nanostructure.

\section{Results and Discussion}

The morphology of the synthesized nanostructures, supported by carbon paper substrate, was initially examined using scanning electron microscopy (SEM), as shown in Figure $1(\mathrm{a}-\mathrm{e})$. The carbon paper substrate consists of small graphitic fibers, 5-10 $\mu \mathrm{m}$ diameter (inset in Figure 1a). A high density of radical nanostructures grown on the graphitic fibers is clearly evident in Figure 1a. The straight and isolated nanostructures, 5-10 $\mu \mathrm{m}$ in length, are aligned perpendicular to the surface of the graphitic fibers (Figure 1b and c). Close examination reveals that a dark region, about $1 \mu \mathrm{m}$ in length, is present on the tips and the bottoms of each nanostructure (Figure 1d and e).

Further characterization of the nanostructures was carried out by using transmission electron microscopy (TEM), as shown in Figure $2 \mathrm{a}-\mathrm{d}$. Again, a relatively light region, about $1 \mu \mathrm{m}$ in length, is clearly evident at the tip of each nanostructure (Figure 2a). Further, light regions of similar length were also observed at the bottom of each individual nanostructure. Scrutiny of the tips of the nanostructures revealed two types of tip structures as indicated by the square and circle in Figure 2a, respectively. Most nanostructures exhibit a small dark region at the end of the tips, separated from the main dark region by the light region, as shown in Figure 2b. Further, nanostructures, without a dark tip end, are also distinguishable (Figure 2c). An increased magnification transmission electron micrograph of the nanostructure revealed a dark core and a thin outer layer of about $30 \mathrm{~nm}$ thick, as shown in the inset of Figure 2d. Energydispersive X-ray (EDX) analysis (see Supporting Information of Figure S1) taken from several fragments of Figure $2 b$ reveals the existence of Sn and $\mathrm{C}$ in the dark regions; however, within the light region, EDX analysis detected mainly carbon with a very low level of tin. This indicates that the light regions are hollow carbon nanotubes, and the dark regions are carbon nanotube encapsulated tin nanowires.

A high-resolution TEM lattice image of the tin nanowire is shown in Figure $2 \mathrm{~d}$. The measured fringe spacing of the nanowire was $0.414 \mathrm{~nm}$, which is in good agreement with the $d$-value of $0.412 \mathrm{~nm}$ for the $\{110\}_{\mathrm{Sn}}$ set of planes, ${ }^{11}$ confirming that nanowires are pure metallic tin. Further, it is evident that the carbon-encapsulating layer is featureless, suggesting amorphous carbon nature. Usually, the amorphous carbon layer covering the nanowires is composed of short-distance order and 


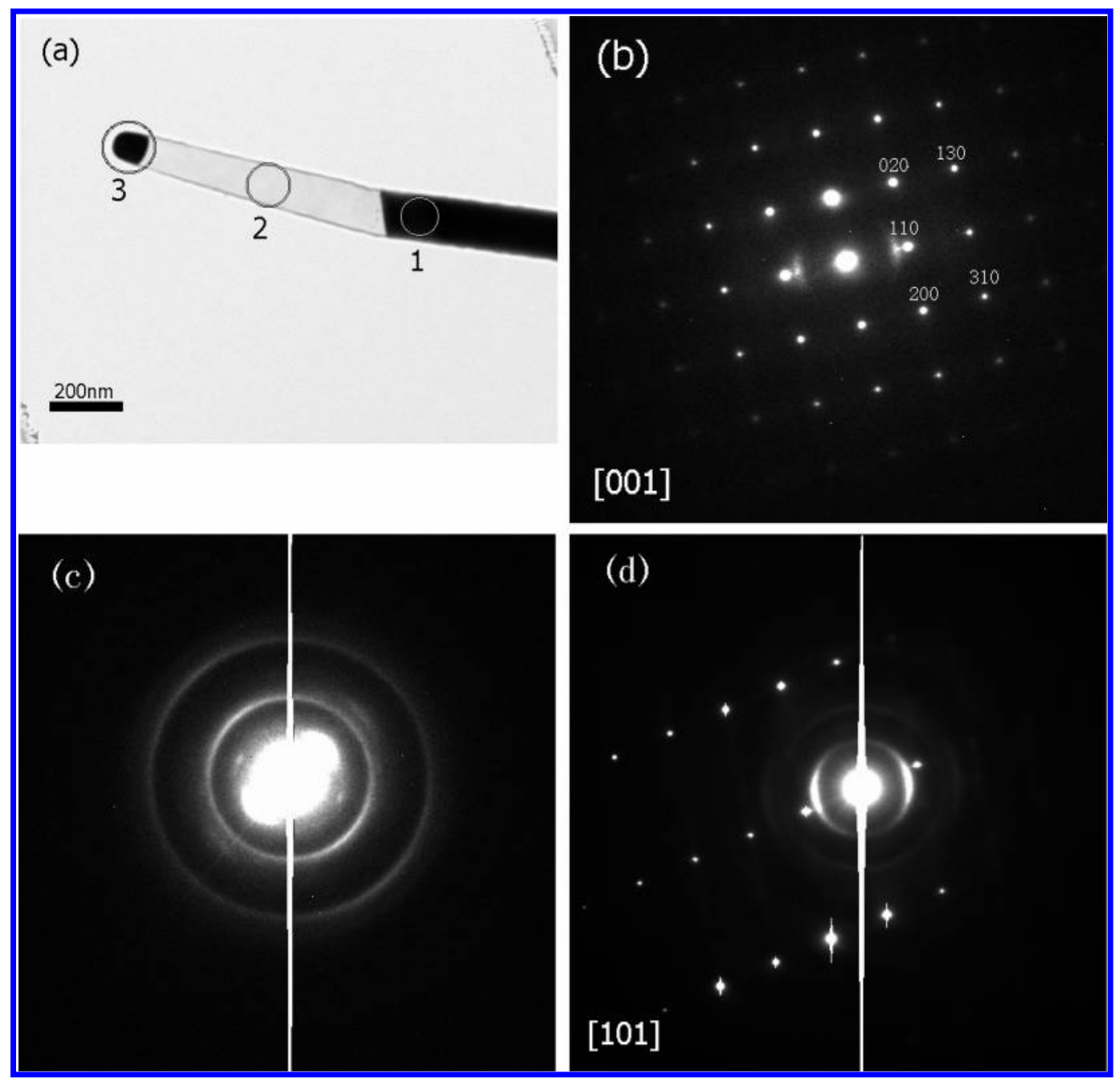

Figure 3. (a) TEM micrograph of an individual a-CNT-Sn; (b-d) the corresponding selected area electron diffraction patterns taken from the bottom region, the CNT hollow region, and the tip of a heterostructure, respectively.

long-distance disorder, as observed for amorphous carbon nanotubes. ${ }^{7 \mathrm{~d}}$ Thus, coaxially grown heterostructures, consisting of metallic tin nanowires and amorphous carbon encapsulating nanotubes, were synthesized in the present study, here named simply as "a-CNT-Sn".

Selected area electron diffraction (SAED) patterns of the a-CNT - Sn heterostructures are shown in Figure 3. The SAED patterns were recorded from the bottom region, the CNT hollow region, and the tip of heterostructures, respectively, as indicated by circles 1, 2, and 3 in Figure 3a. The SAED pattern obtained from the bottom region of the carbon nanotube encapsulated tin nanowires can be fully indexed as a metallic $\beta$-Sn along a [001] zone axis. Further, by recording the SAED pattern along the nanostructure, it was revealed that the tin nanowire in each individual heterostructure is a single crystal. The SAED pattern of the CNT hollow region (Figure 3c) illustrates clearly amorphous diffusion rings. For the tip of the heterostructure (Figure 3d), again, the SAED pattern can be fully indexed as a metallic $\beta$-Sn. Further, diffusion rings, from the amorphous carbon encapsulating nanotubes, were also revealed in Figure $3 \mathrm{~b}$ and $\mathrm{d}$. The structure of single-crystal tin and amorphous carbon was further confirmed by X-ray diffraction (XRD) analysis and Raman spectroscopy, as shown in Figures S2 and S3 of Supporting Information.

The a-CNTs - Sn heterostructures were synthesized through thermal reactions between Sn powder and ethylene. On the basis of the above careful structural analysis, here, a growth mech- anism for a-CNTs $-\mathrm{Sn}$ heterostructures is proposed, as illustrated schematically in Figure 4.

(a) During thermal evaporation at $900{ }^{\circ} \mathrm{C}$, Sn vapor is generated from the molten $\mathrm{Sn}$ powder ( $\mathrm{Sn}$ : melting point $232{ }^{\circ} \mathrm{C}$, boiling point $2270{ }^{\circ} \mathrm{C}^{12}$ ). The $\mathrm{Sn}$ vapor is then transported downstream by the carrier gas (Ar) and then is deposited in the form of liquid droplets on the surface of graphitic fiber substrate. The resultant $\mathrm{Sn}$ liquid droplet provided an energetically favored site for further deposition of in-coming $\mathrm{Sn}$ vapor, resulting in the formation of a bigger Sn liquid droplet on graphitic fiber (Figure 4a).

(b) Two different 1D nanostructure growth processes occurred simultaneously. On one hand, the liquid Sn droplets, acting as catalysts, decomposed ethylene gas on the Sn surface to form carbon nanotubes from the bottom of the Sn droplet (Figure $4 b)$. This growth mechanism of carbon nanotubes involves the diffusion of carbon in the catalytic particle and carbon precipitation at its surface. ${ }^{13}$ Metal $\mathrm{Sn}$ has been previously reported as active cocatalysts for growing carbon nanotubes. ${ }^{14}$ However, compared with the most commonly used catalysts such as Fe, $\mathrm{Co}, \mathrm{Ni}$, and their alloys, the catalytic effectiveness of tin on carbon nanotube growth is lower because of the relatively low carbon solubility in tin. ${ }^{15}$ The relatively low catalytic effectiveness might be responsible for the formation of amorphous carbon nanotubes rather than graphitic carbon nanotubes.

(c) On the other hand, simultaneous to the above process, $\mathrm{Sn}$ becomes supersaturated in the liquid $\mathrm{Sn}-\mathrm{C}$ alloy droplet 


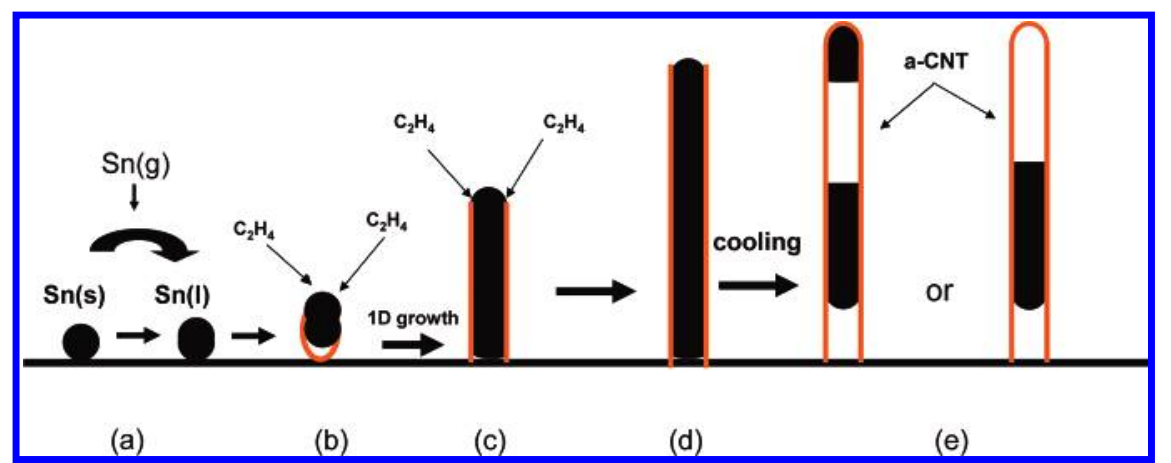

Figure 4. (a-e) Schematic diagram illustrating the growth mechanism of the a-CNT-Sn nanostructures: amorphous carbon nanotube encapsulating single-crystalline Sn nanowires on a graphitic fiber. (a) Sn powder first vaporizes and then condenses to form nuclei of a Sn liquid droplet. (b) Carbon nanotube starts to grow at the bottom of Sn droplet that acts as a catalyst and, meanwhile, the growth of 1D Sn nanowires is initiated by self-catalyzed VLS process. (c) Carbon nanotube and Sn nanowire continuously grow coaxially. (d) Carbon nanotube closes at the end tip when the process is completed. (e) Form either cavity below tip or on head tip.

with the continued absorption of $\mathrm{Sn}$ vapor, and the $\mathrm{Sn}$ atoms segregate from the alloy, yielding the Sn nuclei at the surface of the droplet, which gives rise to the 1D growth of the $\mathrm{Sn}$ wires while the Sn vapor is available (Figure 4c). In fact, the growth of Sn nanowires observed here follows the mechanism of Sn self-catalyzed vapor-liquid-solid (VLS) process for 1D Sn nanowires. ${ }^{16}$

(d) The growth of $\mathrm{Sn}$ nanowire in the central core and the growth of carbon nanotubes in the shell can occur simultaneously, providing the availability of both $\mathrm{Sn}$ and carbon sources. As a result, the continuation of these two processes finally produces heterostructure geometry, as shown in Figure $4 d$.

(e) When the reactor is cooled down, the growth process of Sn nanowires is subsequently finished, and the sheathed carbon nanotubes tend to close the tip end. Upon temperature completely cooled down, the 1D liquid Sn nanowires solidifies and shrinks down because of the difference in volume between the liquid and the solid and the capillary suction within the amorphous carbon nanotubes, ${ }^{17}$ which lead to hollow tube features because of either the partial shrinkage or complete shrinkage of the Sn from the top and bottom of the heterostructure (Figure 4e). Eventually, the two distinct morphologies of amorphous carbon encapsulated single-crystalline Sn nanowires have been finally produced on graphitic fiber. These melting and expansion behaviors of the 1D nanowires inside carbon nanotubes have been reported by several groups, and they are indeed significantly different from that of a macroscopic state. ${ }^{17 \mathrm{~b}, 17 \mathrm{c}}$ The phenomenon of melting/solidifying and moving of Sn filling within the tubes (including carbon tubes) has been observed and reported previously under electron irradiation or during thermal annealing. ${ }^{17 \mathrm{~b}, 17 \mathrm{c}, 17 \mathrm{f}}$

The present method demonstrated several advantages on the production of such a unique heterostructure consisting of metallic Sn nanowires encapsulated in amorphous carbon nanotubes: (1) Unlike the AAM template-based method that uses a two-step process to generate carbon nanotube-nanowire heterostructures, ${ }^{18}$ our present method only involves a one-step "in-situ" procedure, without using any external catalysts. (2) Compared with other common filling techniques such as deposition by capillary action, ${ }^{17 \text { a }}$ wet-chemistry methods, ${ }^{19}$ electrochemical deposition, ${ }^{20}$ arc-discharge plasma, ${ }^{21}$ and condensed-phase electrolysis, ${ }^{22}$ our present method can achieve a very high yield. (3) Our present method allows the growth of high-density heterostructures on various commercially available substrates such as carbon paper and silicon. (4) Our method can provide a compliant surface coating, such as amorphous carbon layer here, which is necessary to enhance nanowirematrix (e.g., polymer) interfacial bonding strength. (5) The presence of a-CNTs, as a protective sheath (or coating) in our method, can stabilize metal nanowires inside and prevent them from oxidation. Therefore, it is expected that such heterostructures, consisting of a-CNTs and metal nanowires, will exhibit higher mechanical properties, ${ }^{23}$ enhanced electrical properties, ${ }^{3 \mathrm{~d}}$ higher corrosion and oxidation resistance, higher Li-ion storage capacities, ${ }^{4 c, 24}$ and better composite properties when combined with other materials.

\section{Conclusions}

We have demonstrated the first synthesis of heterostructures of amorphous carbon nanotubes encapsulated single-crystalline tin nanowires (a-CNT-Sn) on micrographite fibers by employing the simple chemical vapor deposition under an atmosphere of argon/ethylene at $900{ }^{\circ} \mathrm{C}$. The well-defined tin nanowires, 200-400 $\mathrm{nm}$ in diameter, are completely covered with amorphous carbon walls of $30 \mathrm{~nm}$ thickness. A growth mechanism has been proposed to explain the simultaneous growth of amorphous carbon nanotubes and tin nanowires. The high yield fabrication of a-CNT-Sn heterostructures will permit further exploration of their properties and potential applications. The use of commercially available carbon paper as substrate for the synthesis of such heterostructures is of significant importance to potential industrial applications in the areas of sensors and fuel cells.

Acknowledgment. This research was supported by General Motors of Canada, Natural Sciences and Engineering Research Council of Canada (NSERC), Canada Foundation for Innovation (CFI), Ontario Early Researcher Award (ERA), and the University of Western Ontario (Academic Development Fund (ADF) and the Start-up Fund). Dr. Xiangcheng Sun thanks visiting fellowship from Centre for Chemical Physics, The University of Western Ontario. We also thank Dr. Fengping Wang and Dr. Zhifeng Ding for Raman analysis.

Supporting Information Available: Energy-dispersive Xray (EDX) and Raman and XRD spectra of the nanostructures are shown in Figures S1, S2, and S3, respectively. This material is available free of charge via the Internet at http://pubs.acs.org.

\section{References and Notes}

(1) (a) Iijima, S. Nature 1991, 354, 56. (b) Baughman, R. H.; Zakhidov, A. A.; Heer, W. A. Science 2002, 297, 787. (c) Beguin, F., Ehrburger, P., Eds. Special issue on carbon nanotubes in Carbon 2002, 40, 1619. (d) 
Haddon, R. C., Ed. Special issue on carbon nanotubes in Acc. Chem. Res. 2002, 35, 997. (e) Dresselhaus, M. S., Dai, H., Eds. Special issue on carbon nanotubes in MRS Bull. 2004, 29, 237.

(2) Xia, Y.; Yang, P.; Sun, Y.; Wu, Y.; Mayers, B.; Gates, B.; Yin, T.; Kim, F.; Yan, H. Adv. Mater. 2003, 15, 353.

(3) (a) Hu, J.; Bando, Y.; Zhan, J.; Zhi, C.; Golberg, D. Nano Lett. 2006, 6, 1136. (b) Rana, R. K.; Pol, V. G.; Felner, I.; Meridor, E.; Frydman, A.; Gedanken, A. Adv. Mater. 2004, 16, 972. (c) Jankovic, L.; Gournis, D.; Trikalitis, P. N.; Arfaoui, I.; Cren, T.; Rudolf, P.; Sage, M. H.; Kooi, T. T. M. B.; Hosson, J. D.; Karakassides, M. A.; Dimos, K.; Moukarika, A.; Bakas, T. Nano Lett. 2006, 6, 1131. (d) Han, W.; Zettl, A. Adv. Mater 2002, 14, 1560

(4) (a) Luo, L.; Huang, Z. P.; Zhao, Y. G.; Zhang, L.; Zhu, J. Adv. Mater. 2004, 16, 1512. (b) Bao, J.; Tie, C.; Xu, Z.; Suo, Z.; Zhou, Q.; Hong, J. Adv. Mater. 2002, 14, 1483. (c) Wang, Y.; Zeng, H. C.; Lee, J. Y. Adv. Mater. 2006, 18, 645. (d) Luo, J.; Xing, Y.; Zhu, J.; Yu, D. P.; Zhao, Y. G.; Zhang, L.; Fang, H.; Huang, Z. P.; Xu, J. Adv. Funct. Mater. 2006, 16, 1081.

(5) (a) Liu, J. W.; Li, X. J.; Dai, L. M. Adv. Mater. 2006, 18, 1740. (b) Hu, J.; Ouyang, M.; Yang, P.; Lieber, C. M. Nature 1999, 399, 48. (c) Du, J.; Fu, L.; Liu, Z.; Han, B.; Li, Z.; Liu, Y.; Sun, Z.; Zhu, D. J. Phvs. Chem. B 2005, 109, 12772. (d) Wei, D.; Cao, L.; Fu, L.; Li, X.; Wang, Y.; Yu, C.; Liu, Y. Adv. Mater. 2007, 19, 386. 1096.

(6) Sun, Y. P.; Fu, K.; Lin, Y.; Huang, W. Acc. Chem. Res. 2002, 35 ,

(7) (a) Nishino, H.; Yamaguchi, C.; Nakaoka, H.; Nishida, R. Carbon 2003, 41, 2159. (b) Zhao, N. Q.; He, C. N.; Du, X. W.; Shi, C. S.; Li, J. J. Cui, L. Carbon 2006, 44, 1845. (c) Zhao, T. K.; Liu, Y. N.; Zhu, J. W. Carbon 2005, 43, 2907. (d) Hu, Z. D.; Hu, Y. F.; Chen, Q.; Duan, X. F.; Peng, L. M. J. Phvs. Chem. B 2006, 110, 8263.

(8) (a) Wang, Y.; Su, F.; Lee, J. Y.; Zhao, X. S. Chem. Mater. 2006, 18, 1347. (b) Lee, K. T.; Jung, S. Y.; Oh, S. M. J. Am. Chem. Soc. 2003 , 125,5652

(9) (a) Sun, S. H.; Meng, G. W.; Zhang, M. G.; An, X. H.; Wu, G. S.; Zhang, L. D. J. Phys. D: Appl. Phvs. 2004, 37, 409-412. (b) Hu, J. Q.; Ma, X. L.; Shang, N. G.; Xie, Z. Y.; Wong, N. B.; Lee, C. S.; Lee, S. T. J. Phvs. Chem. B 2002, 106, 3823-3826. (c) Luo, S.; Fan, J.; Liu, W.; Zhang, M.; Song, Z.; Lin, C.; Wu, X.; Chu, P. Nanotechnologv 2006, 17 1695-1699. (d) Yang, R.; Wang, L. Z. J. Am. Chem. Soc. 2006, 128, 14661467.
(10) Zhang, J.; Yang, Y.; Jiang, F.; Xu, B.; Li, J.; Wang, X.; Wang, S. Nanotechnology 2005, 16, 2887.

(11) (a) Deshpande, V. T.; Sirdeshmukh, D. B. Acta Crvstallogr. 1961, 14, 355. (b) Hsu, Y. J.; Lu, S. Y. J. Phys. Chem. 2005, 109, 4398.

(12) Lange's Handbook of Chemistry, 15th ed.; Dean, J. A., Ed.; McGraw-Hill: New York, 1999.

(13) (a) Sun, X.; Stansfield, B.; Dodelet, J. P.; Désilets, S. Chem. Phvs. Lett. 2002, 363, 415. (b) Sun, X.; Li, R.; Stansfield, B.; Dodelet, J. P.; Désilets, S. Carbon 2007, 45, 732.

(14) (a) Okazaki, N.; Hosokawa, S.; Goto, T.; Nakayama, Y. J. Phvs. Chem. B 2005, 9, 17366. (b) Pan, L.; Hayashida, T.; Harada, A.; Nakayama, Y. Phvsica B 2002, 323, 350

(15) Moisala, A.; Nasibulin, A. G.; Kauppinen, E. I. J. Phys.: Condens. Matter 2003, 15, S3011.

(16) (a) Orlandi, M. O.; Leite, E. R.; Aguiar, R.; Bettini, J.; Longo, E. J. Phvs. Chem. B 2006, 110, 6621. (b) Chen, Y. X.; Campbell, L. J.; Zhou, W. L. J. Crust. Growth 2004, 270, 505.

(17) (a) Ajayan, P. M.; Iijima, S. Nature 1993, 361, 333. (b) Hsu, W K.; Terrones, M.; Terrones, H.; Grobert, N.; Kirkland, A. I.; Hare, J. P.; Prassides, K.; Townsend, P. D.; Kroto, H. W.; Walton, D. R. M. Chem. Phvs. Lett. 1998, 284, 177. (c) Wu, Y. Y.; Yang, P. D. Adv. Mater. 2001, 13, 520. (d) Gao, Y.; Bando, Y.; Golberg, D. Appl. Phys. Lett. 2002, 81 , 4133. (e) Gao, Y.; Bando, Y. Nature 2002, 415, 600. (f) Hu, J.; Bando, Y.; Zhu, J.; Golberg, D. Angew. Chem., Int. Ed. 2004, 43, 4606.

(18) (a) Luo, J.; Zhang, L.; Zhang, Y.; Zhou, J. Adv. Mater. 2002, 12, 1413. (b) Bao, J.; Tie, C.; Xu, Z.; Suo, Z.; Zhou, Q.; Hong, J. Adv. Mater. 2002, 14, 1483

(19) (a) Tsang, S. C.; Chen, Y. K.; Harris, P. J. F.; Green, M. L. H. Nature 1994, 372, 159. (b) Hsin, Y. L.; Hwang, K. C.; Chen, F. R.; Kai, J. J.; Adv. Mater. 2001, 13, 830

(20) Wang, Y.; Zeng, H. C.; Lee, J. Y. Adv. Mater. 2006, 18, 645.

(21) (a) Tsang, S. C.; Chen, Y. K.; Harris, P. J. F.; Green, M. L. H Nature 1994, 372, 159. (b) Hsin, Y. L.; Hwang, K. C.; Chen, F. R.; Kai, J. J.Adv. Mater. 2001, 13, 830.

(22) Hsu, W. K.; Hare, J. P.; Terrones, M.; Harris, P. J. F.; Kroto, H. W.; Walton, D. R. M. Nature 1995, 377, 687.

(23) Yang, W.; Araki, H.; Tang, C.; Thaveethavorn, S.; Kohyama, A.; Suzuki, H.; Noda, T. Adv. Mater. 2005, 17, 1519.

(24) Kumar, T. P.; Ramesh, R.; Lin, Y. Y.; Fey, G. T. K. Electrochem. Commun. 2004, 6, 520-525. 